\title{
Pseudo-aneurysms in relationship to massive cerebral haemorrhage
}

\author{
F. M. COLE AND P. O. YATES \\ From the Department of Pathology, University of Manchester
}

This paper deals with two types of small body which are found within or close to a massive intracerebral haemorrhage. These bodies have, in the past, been confused with true aneurysms (Charcot and Bouchard, 1868), and for many years it was generally accepted that they were causally related to the main haemorrhage.

In 1909 Ellis examined many of these structures from 34 brains with cerebral haemorrhage and concluded that they were not dilatations of arteries but were in fact nothing more than perivascular collections of blood clot which had escaped from small vessels by simple rupture of the wall or by a gradual dissection of the layers. Nevertheless, he believed that these blood clots or 'false' aneurysms were formed before the large haemorrhage and did themselves rupture to give rise to it. Although the latter view was not accepted, this denial of the existence of true aneurysms resulted in subsequent workers seeking alternative mechanisms for this type of stroke, the aneurysmal theory of cerebral haemorrhage being almost entirely abandoned for many years. The 'false' aneurysms were also largely forgotten, and only passing reference to them has been made in the literature since that time. They have been described as dissecting aneurysms (Globus and Epstein, 1952), unorganized arteriolar haematomata (Adams and Vander Eecken, 1953) and perivascular collections of blood (Russell, 1954).

The picture was somewhat complicated by the fact that, following a painstaking histological survey of 10 brains, Green had in 1930 described three true aneurysms, that is, localized dilatations of intracerebral arteries. This was the first unquestionable description of such lesions, but its real significance did not become apparent for many years. Blackwood (1963), for example, felt that Green's lesions were identical to those described by Charcot and Bouchard. It was not until 33 years after Green's discovery that, by using the much more efficient technique of post-mortem angiography, the presence of these true aneurysms was confirmed and their real numbers described (Ross Russell, 1963). It was during the course of a similar study of the incidence of these intracerebral aneurysms in 200 subjects (Cole and Yates, 1967) that the present observations were made on the nature and mode of formation of the small bodies or 'false' aneurysms of the early workers.

\section{METHODS}

Twelve brains showing massive haemorrhage of the classical type were studied. In all cases the haemorrhage had occurred into the lenticulo-striate areas. All the subjects were more than 60 years old, and all were known to suffer from hypertension. (Diastolic blood pressure over $110 \mathrm{~mm} . \mathrm{Hg}$ and heart weight over $400 \mathrm{~g}$. for a man or $350 \mathrm{~g}$. for a woman.)

Ten brains were examined in which intracerebral haemorrhage was not associated with hypertension; in seven cases it followed rupture of an aneurysm of the circle of Willis; three were cases of leukaemia and one of idiopathic thrombocytopenia.

The brains were examined in a way similar to that employed by early workers (Charcot and Bouchard, 1868). Portions of brain tissue adjacent to the haemorrhage were immersed in a stream of running water and the vessels were inspected when they had separated from the surrounding parenchyma. Other portions and those which included vascular bodies were fixed and examined histologically. Using this relatively crude technique two types of structure can be identified.

\section{FINDINGS}

TYPE I STRUCTURES (FIG. 1) These are found in the walls of the haemorrhagic cavity and within the haematoma itself. They separate quite easily from the brain tissue under water and measure from $1 \mathrm{~mm}$. to $1 \mathrm{~cm}$. in diameter. They are firm, dark red and appear as either uniform swellings surrounding a blood vessel or eccentrically placed sacs with the vessel attached to one pole. Frequently they form terminal bulbous enlargements on the end of a torn vessel. None is seen showing evidence of what might be regarded as rupture of its wall, of the kind which Ellis had suggested could lead to a large haemorrhage. The numbers per brain vary from one to $\mathbf{1 0}$ for those visible to the naked eye, but many 


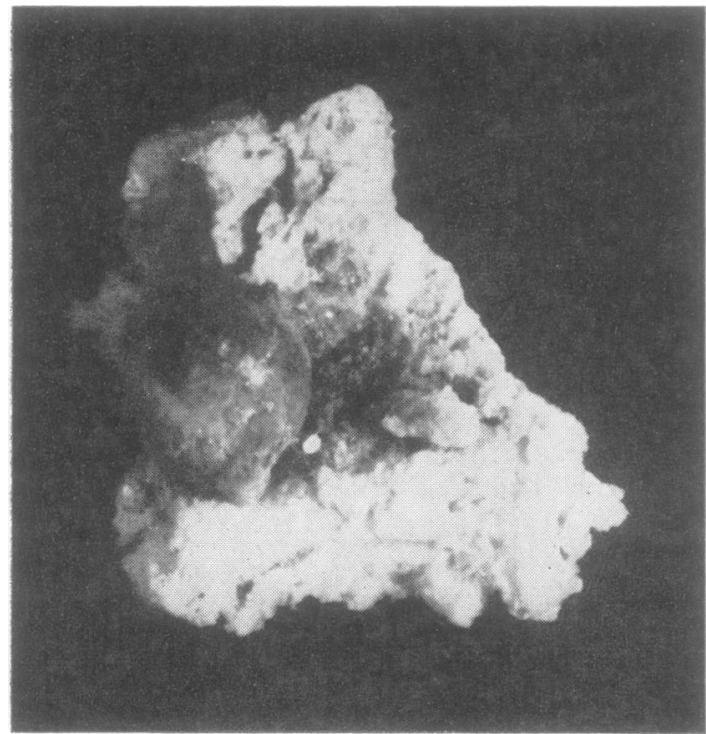

FIG. 1. A type I structure in the wall of a haemorrhagic cavity $(\times 3)$.

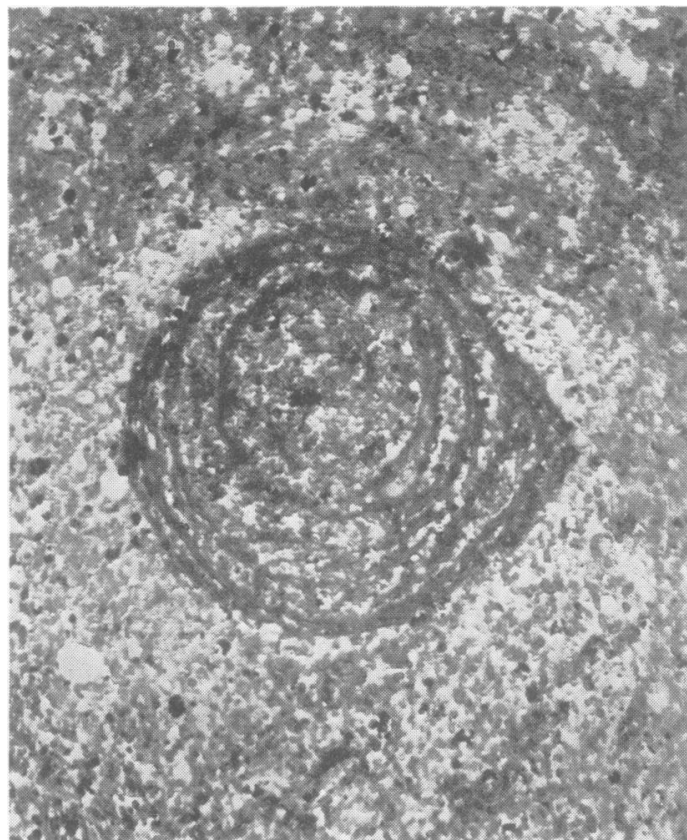
FIG. 3. Another type I lesion sectioned at right angles to $\stackrel{\mathrm{D}}{\mathrm{O}}$ 으 the longitudinal axis of the parent artery. No connexion $\stackrel{\vec{D}}{\vec{D}}$ with the vessel can be seen (haematoxylin and eosin $\times 40$ ). $\varrho \vec{~}$

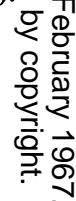

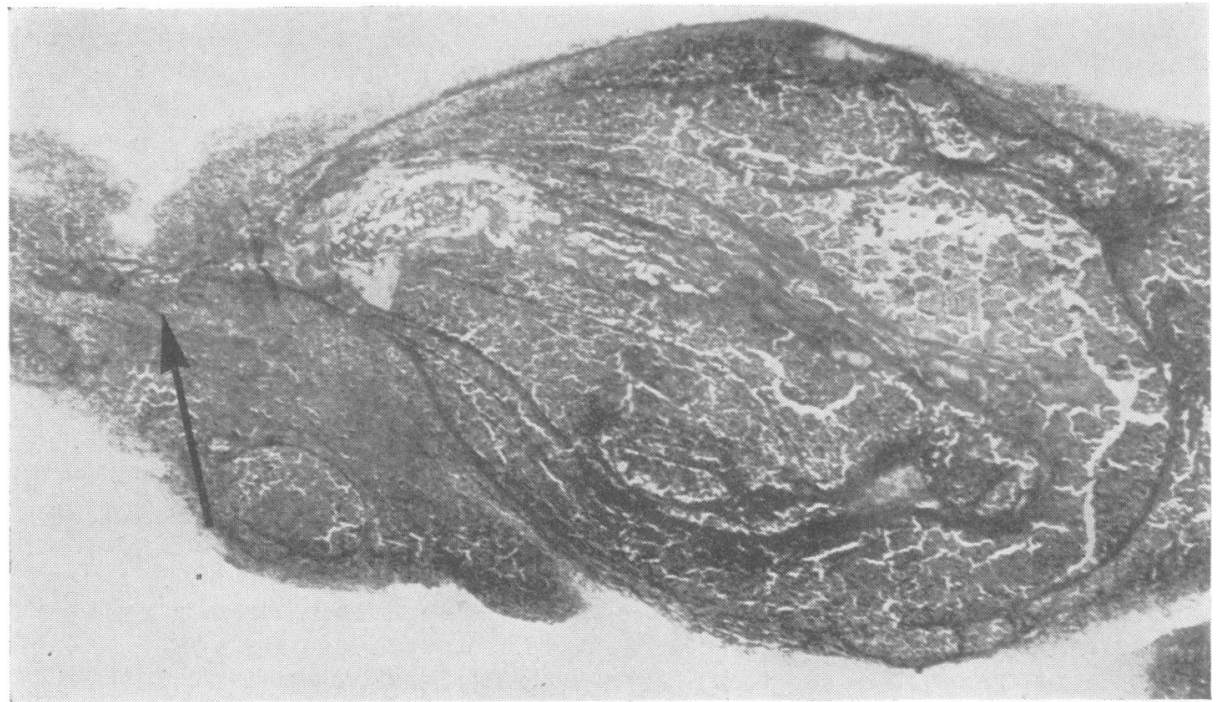

FIG. 2. A type I lesion in longitudinal section. The arrow indicates the small arteriole of origin (haematoxylin and eosin $\times 7$ ). 
more can be found on microscopic examination. All are found within or in close relationship to the main haemorrhage.

Microscopy Sometimes sections clearly show the connexion with a vessel (Fig. 2) but this is not the case if the plane of section passes through the body at $90^{\circ}$ to the longitudinal axis of the vessel (Fig. 3). The walls of type I bodies are made up of bands or sheets of fibrin arranged in a laminated fashion and between these concentric layers red cells and polymorphs are found. The outer fibrin layer sharply separates the brain tissue from the contents of the body, and it is this layer which acts as a capsule and allows easy separation during maceration. It is quite evident that the whole structure is due to a peculiar form of extravascular blood coagulation. There is nothing to suggest that it represents a dilatation of the parent vessel and there is therefore no question of this type of structure being an aneurysm of any type, true or dissecting. In every case in which the parent vessel is identifiable as it runs into the lesion it proves to be an arteriole or small artery (Fig. 2). The surrounding tissue shows only acute reactionary change indicating that the lesions occur shortly before, during, or after the main haemorrhage.

TYPE II STRUCTURES (FIG. 4) These lesions occur very much more frequently than type I. They are also found in the wall of the haemorrhagic focus but are much smaller, rarely being more than $1 \mathrm{~mm}$. in diameter. They present as a series of bubble-like swellings of blood along the long axis of the small vessels and are in direct continuity with the main mass of haemorrhage. Examination with a dissecting microscope using transmitted light shows quite clearly that they consist of collections of fluid blood which have assumed spherical or fusiform shapes within the perivascular space, and are limited by the perivascular membrane. When viewed with incident light they present an appearance strikingly similar to small aneurysms. Histologically (Fig. 5) this type of lesion must be sought carefully as a section passing through a sufficient length of vessel exactly in the longitudinal axis is not often seen.

\section{DISCUSSION}

RELATIONSHIP BETWEEN THESE LESIONS AND THOSE DESCRIBED BY EARLIER WORKERS If the texts and illustrations of the work of Charcot and Bouchard are compared with the verbal descriptions in Ellis's paper (no illustrations were provided) two things are apparent. First it is clear that Ellis was correct in his opinion that the lesions he described were not aneurysms, and secondly that there are several major discrepancies between the two accounts.

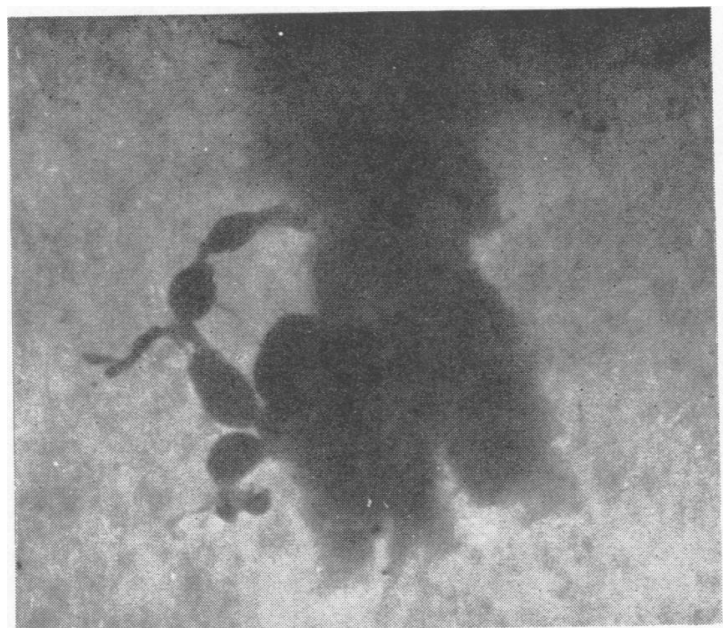

FIG. 4. Type II lesions as seen by transmitted light. Note the 'string of beads' appearance. The mass on the left of the bodies is a mass of brain tissue $(\times 6)$.

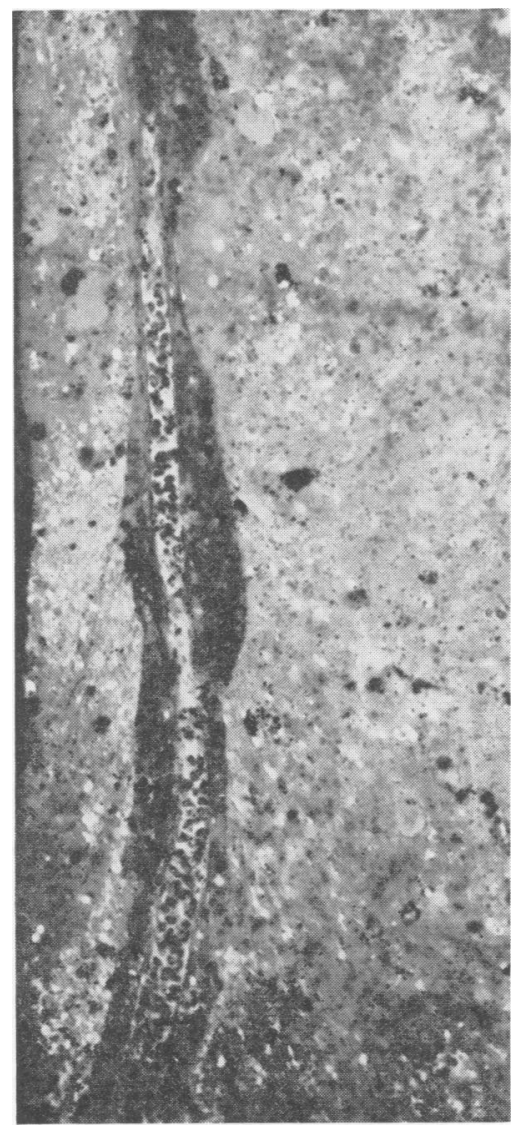

FIG. 5 .

Histological appearance of type II lesion showing the perivascular space distended by blood (haematoxylin and eosin $\times 30$ ). 

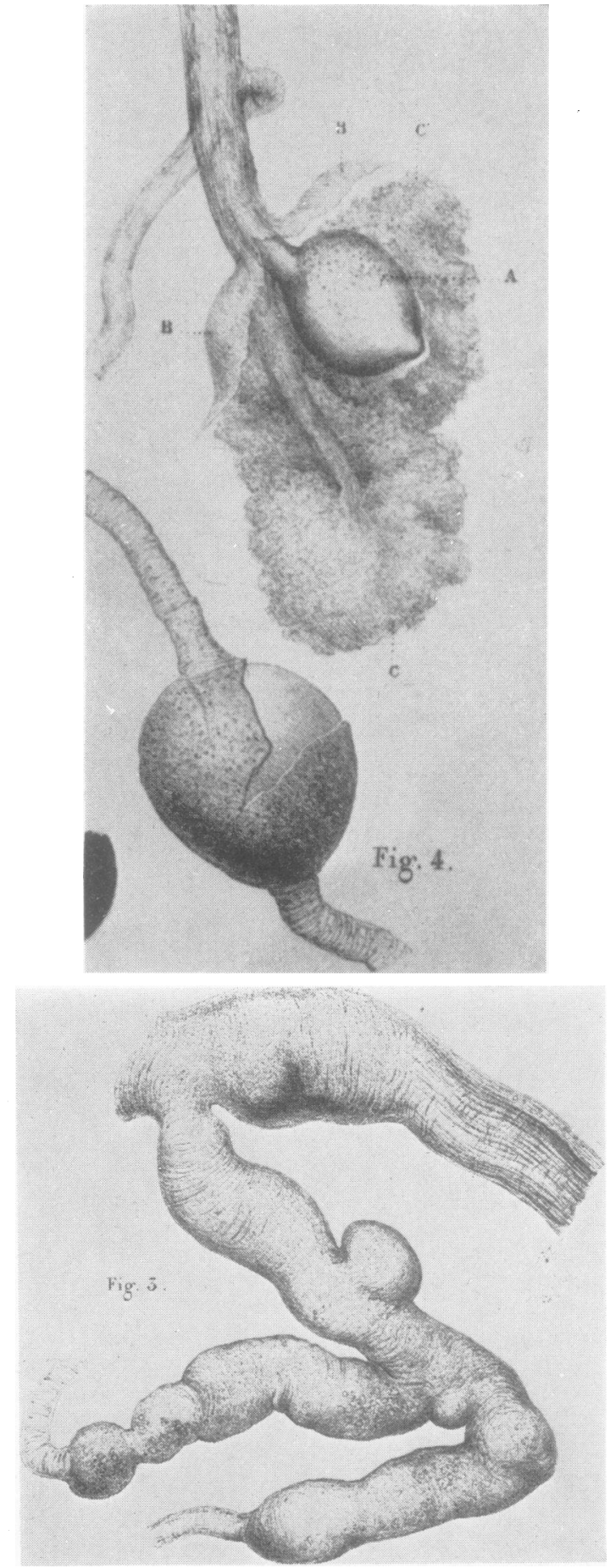

FIGS. 6 and 7. Illustrations of 'aneurysms' from Charcot and Bouchard's article. Note the difference in the two types of lesion.
1 The Ellis lesions are much larger. Ellis himself recognized this discrepancy and called his lesions 'supermiliary' as opposed to Charcot and Bouchard's 'miliary' ones. Whereas the latter lesions were no larger than $1 \mathrm{~mm}$. in diameter, some of those found by Ellis were as big as $1 \mathrm{~cm}$.

2 The numbers found in each brain differed, Charcot and Bouchard finding as many as 200 with the naked eye, whilst Ellis found up to 20 .

3 The shape and relationship to vessels of the majority of the Charcot-Bouchard bodies differed from those described by Ellis. Figures 6 and 7 are illustrations from Charcot and Bouchard's original paper. Figure 6 corresponds exactly to Ellis's description of his lesions and also to the present type I (Fig. 1). However, the type illustrated in Fig. 7 was not described by Ellis, although it was in this form that Charcot found the majority of his 'aneurysms'. This type is in the present paper referred to as type II (Fig. 4).

This failure of Ellis to recognize the two types of body which Charcot and Bouchard described accounts for the discrepancy of numbers, size, and configuration.

SIGNIFICANCE OF THE TWO TYPES OF HAEMATOMA Both Ellis and Charcot and Bouchard considered that the lesions which they described were $(a)$ specific for the classical type of hypertensive haemorrhage; $(b)$ the result of degenerative vascular changes (Charcoto and Bouchard stated that periarteritis was responsible, and Ellis felt that arteriosclerosis was the primary cause); and (c) that they could rupture to release a massive haemorrhage. Neither made mention of the possibility that the structures which they described might be the result, and not the cause, of the large haemorrhage.

With this possibility in mind and to determine just how specific both type I and type II lesions are in hypertensive haemorrhage, it was decided to examine brains showing massive intracerebral haemorrhage which had occurred in young people (less than 40 years of age); those with no evidence of generalized vascular disease or hypertension who had suffered haemorrhage as a result of conditions other than hypertension.

In all 10 cases, either by maceration or microscopy of the fresh or fixed material, both type I and type II lesions were found. Indeed Fig. 8 shows the type I structures isolated from a case of intracerebral haemorrhage due to rupture of a berry aneurysm of the circle of Willis occuring in a young man. It seems then that neither lesion can be considered at all specific for hypertensive cerebral haemorrhage and can hardly be regarded as having any causal relationship to it. 


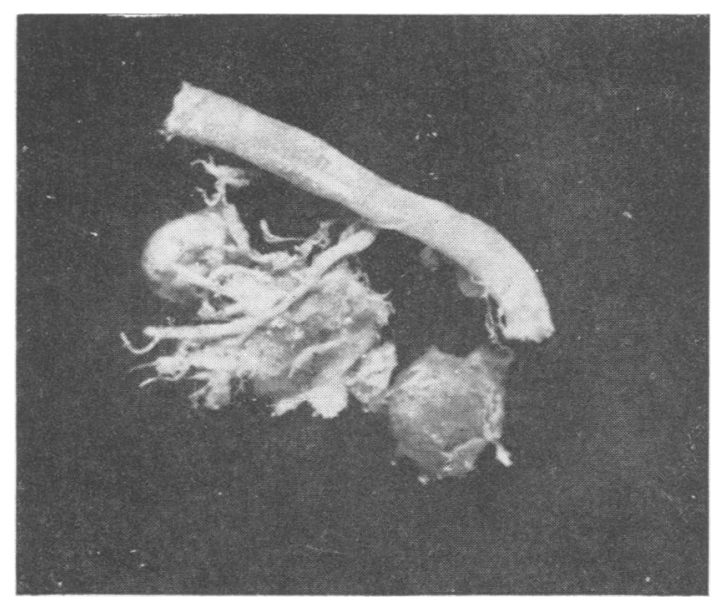

FIG. 8. Small artery bearing three type I lesions from a case of intracerebral haemorrhage due to rupture of a berry aneurysm of the circle of Willis in a young man $(\times 4)$.

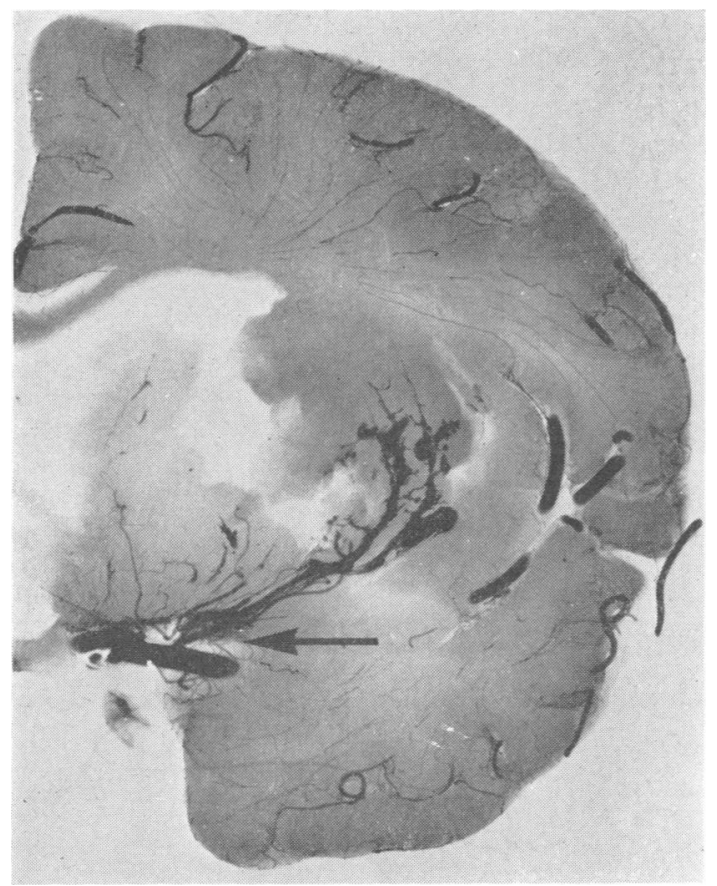

FIG. 9. Radiograph (positive print) of a coronal section of brain with cerebral haemorrhage. Note the displacement of the striate arteries to the right and the narrow angle which they form with the middle cerebral artery (arrow).
This finding that pseudo-aneurysms are common in people under the age of $\mathbf{4 0}$ having a large cerebral haemorrhage contrasts with the extreme rarity of true aneurysms under this age (Cole and Yates, 1967).

THE ORIGIN OF THESE LESIONS The 10 cases of nonhypertensive haemorrhage in which these lesions were found have, in common with cerebral haemorrhage of the hypertensive variety, one outstanding feature, namely, the presence of a massive, rapidly expanding, space-occupying lesion occurring within brain substance. This lesion must invariably produce acute shift and distortion of adjacent structures. Such a movement is recognized by radiologists in life, and the signs of its presence are of use in differentiating between haemorrhage and infarction (Wechsler and Gross, 1949). More recently an echoencephalographic method has also been used to demonstrate this feature (Achar, Coe, and Marshall, 1966). Shift is also well illustrated in such cases by post-mortem injections of radio-opaque mixtures (Fig. 9). Those striate vessels which lie lateral to the haematoma are displaced even further laterally, and the angle between the middle cerebral artery and the striate vessels is greatly reduced.

Furthermore it can be shown that the small arteries and arterioles close to a large haematoma have ruptured (Fig. 9). The difficulty lies in ensuring that such leaks are not an artefact due to rupture by the pressure of the injection media; they occur, however, when the pressure of injection is reduced to quite low levels $(40 \mathrm{~mm}$. $\mathrm{Hg})$. A similar post-mortem angiographic technique was used to demonstrate that arterial rupture was the basis for secondary brainstem haemorrhages (Johnson and Yates, 1956).

TYPE II LESION The nature of these structures suggests that they are formed in a slightly different way. The blood within the main haematoma is under pressure and tends to escape along the lines of least resistance. The blood projects into the surrounding tissue, separating white matter tracts and fibres with little actual destruction of tissue. Another line of diminished resistance is along the spaces or potential spaces which surround the vessels. It is believed that the 'string of beads' appearance which characterizes type II lesions is accounted for in this way. Again a similar (artificially produced) picture is seen when injection medium escapes from a small vessel and tracks along the perivascular space. This is well illustrated in Fig. 10 and the similarity to an actual type II lesion (Fig. 4) is striking.

The regular beaded form of this lesion is presumably a reflection either of the actual structure of the space or of the physical properties of a narrow 


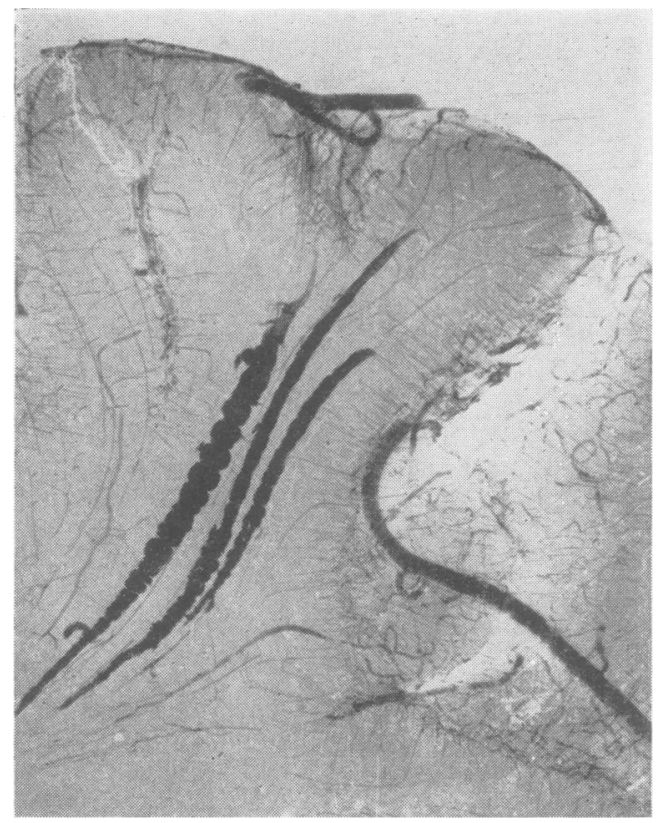

FIG. 10. Radiograph (positive print $\times 4$ ) showing leakage of the injection medium along the perivascular space. The appearance is similar to that seen when blood escapes (Fig. 4).

column of liquid blood confined within an elastic tube. There have been many detailed studies of the anatomy of the perivascular space (Weed, 1914; Woollam and Millen, 1954). None indicates any way in which its structure could have this effect. In a situation of this kind where a narrow liquid cylinder is held within an elastic boundary it is likely to take an unduloid form, even if it does not completely break up into droplets. According to Plateau (1867) the theoretical limit of stability is reached when the length of a liquid cylinder is at least equal to its circumference. However, with increasing viscosity this instability is less, and the unduloid shape may not be apparent until a greater length of liquid is formed, nor will the individual droplets be so spherical. Such a difference in form was indeed noted between the type II structures consisting of blood and those which were produced artificially by the more viscous gelatine injection medium.

\section{SUMMARY}

Attention is drawn to the several different aneurysm- like bodies found in the brain in cases of cerebral haemorrhage.

Some are dilatations of the vascular lumen and therefore true aneurysms. There are two types of pseudo-aneurysmal lesion. Type I, of which 10-20 may be found in each brain, consists of perivascular blood clot, the outer surface being a smooth layer of fibrin. Type II is more common and appears as a beaded distension of the perivascular space by blood.

Neither lesion is specific for, or causally related to, hypertensive cerebral haemorrhage as was previously thought. The mechanisms by which these bodies are formed is suggested.

We are grateful to the technical staff of the Department of Radiology at the Manchester Royal Infirmary for processing the $x$-ray films, Mrs. Celia Worsley for the preparation of the histological material, Mrs. Joan Cornwell for the secretarial work, and the Board of Governors of the United Manchester Hospitals for generous financial assistance.

\section{REFERENCES}

Achar, V. S., Coe, R. P. K., and Marshall, J. (1966). Echoencephalography in the differential diagnosis of cerebral haemorrhage and infarction. Lancet, 1, 161-163.

Adams, R. D. and Vander Eecken, H. M. (1953). Vascular diseases of the brain. Ann. Rev. Med., 4, 213-252.

Blackwood, W. (1963). In Greenfield's Neuropathology by Blackwood, W., McMenemey, W. H., Meyer, A., Norman, R. M., and Russell, D. S., 2nd ed., p. 123. Arnold, London.

Charcot, J. -M., and Bouchard, C. (1868). Nouvelles recherches sur la pathogénie de l'hémorrhagie cérébrale. Arch. Physiol. Norm. Path., 1, 110-127, 643-665.

Cole, F. M., and Yates, P. O. (1967). The occurrence and significance of intracerebral microaneurysms. J. Path. Bact. In press.

Ellis, A. G. (1909). The pathogenesis of spontaneous cerebral haemorrhage. Proc. Path. Soc. Philad., n.s. 12, 197-235.

Globus, J H. and Epstein, J. A. (1952). Massive cerebral haemorrhage: spontaneous and experimentally induced. Proc. 1st. Internat. Congr. Neuropath., Rome, 1952, vol. 1, 289-320.

Green, F. H. K. (1930). Miliary aneurysms in the brain. J. Path. Bact., 33, 71-77.

Johnson, R. T. and Yates, P. O. (1956). Brain stem haemorrhages in expanding supratentorial conditions. Acta radiol. (Stockh.), 46, 250-256.

Plateau, F. (1867). On the spontaneous change of a liquid cylinder into isolated spheres. Lond. Edinb. and Dublin Phil. Mag., 4 ser., 67, 246-248. (Quoted by D'Arcy Wentworth Thompson in On Growth and Form, p. 379. Cambs. Univ. Press, 1942, London).

Russell, Dorothy S. (1954). In Discussion: The pathology of spontaneous intracranial haemorrhage. Proc. roy Soc. Med., 47, 689-704.

Russell, R. W. Ross (1963). Observations on intracerebral aneurysms. Brain, 86, 425-442.

Wechsler, I. S., and Gross, S. W. (1949). Arteriography in cerebral vascular accidents. J. Amer. med. Ass., 139, 502-505.

Weed, L. H. (1914). Studies on cerebro-spinal fluid. Nos. II, III and IV. J. med. Res., n.s. 26, 21-117.

Woollam, D. H. M., and Millen, J. W. (1954). Perivascular spaces of the mammalian central nervous system. Biol. Rev., 29, 251-283. 